\title{
Mathematical Model of Magnetic System of Synchronous Machines with Permanent Magnets and Fractional Single-Toothed Winding
}

\author{
A. F. Shevchenko \\ Department of Mechatronics and Automation \\ Novosibirsk State Technical University \\ Novosibirsk, Russia \\ elmotron@sibmail.ru
}

\begin{abstract}
The article considers the mathematical model for calculating the magnetic circuit of a multipolar synchronous motor with fractional single-toothed winding and permanent magnets. The mathematical model is based on the technique of the tooth contours conductivity. The obtained model makes it possible to calculate these electric machines in steady-state and transient modes, taking into account the discrete structure of the tooth zone and the non-sinusoidal shape of the currents.
\end{abstract}

Keywords- permanent magnets, single-tooth winding, mathematical model

\section{INTRODUCTION}

Multipole synchronous electric machines with fractional single-toothed windings with permanent magnets are used as generators of gearless wind power stations and in contactless controlled electric drives [1,9]. A synchronous generator usually operates at the semiconductor rectifier, but a motor from the static converter of frequency. In both cases, currents with a form far different from sine wave can flow through the synchronous machine windings.

In the machine under investigation with fractional singletoothed windings, magnetic field in the air gap is also far from sinusoidal shape and contains a whole series of the substantially shown higher and lower harmonics, which should be taken into account in the magnetic circuit calculating. These circumstances do not make it possible to use such methods of computation as the method of harmonic conductivities, the grapho-analytical method and etc. to analyze the machines.

It is also problematic to use the numerical techniques for calculating the magnetic field in the machines with fractional single-tooth windings during the whole period of the winding repeatability. A complex shape and a large size of the calculated area within the magnetic field period lead to an increase of the calculation time even for one rotor position with respect to the stator. The need for recurring repetition of such calculations in determining of the dependence of the flux linkage variation on the rotor angular position makes the practical implementation of the method impossible.
For the reasons listed above, the mathematical model of an electric machine with permanent magnets with fractional single-tooth windings is based on the method of conductivity of tooth contours developed by a number of authors at Moscow Power Engineering Institute under direction of A.V. Ivanov-Smolensky [2]. This method allows taking into account simultaneously the gearing of the stator and the rotor, the change in the configuration of the gap when the rotor rotates, the saturation of the magnetic circuit, and also the actual shape of the current in the windings.

The idea of the method of the tooth contours conductivities consists in splitting the two-dimensional region of the active zone into a number of smaller subregions, in each of which the field can be calculated in a linear approximation by the methods of the magnetic field theory [3]. A zone of the magnetic field of the so-called tooth contour is chosen as such subregion, i.e. the contour covering one tooth of the core is used. For the machines under investigation with fractional single-toothed windings with $\mathrm{q}<1$, single-tooth coils are actually tooth contours.

The tooth contour field is calculated under special boundary conditions. The combination of magnetic fields from the currents of the tooth contours gives in the total the magnetic field in the entire active zone.

Thus, in the method of the tooth contours conductivities, the total field of the machine is considered, taking into account its variation in time at the mutual displacement of the stator and the rotor, and not of the individual harmonic components of this field.

The purpose of the article is to develop a mathematical model for the electromagnetic calculation of synchronous machines with single-tooth fractional windings with permanent magnets using the method of the tooth contours conductivity.

\section{THE EQUIVALENT SCHEME OF THE MAGNETIC CIRCUIT}

Fig. 1 shows the equivalent scheme of the magnetic circuit of electric machines with fractional windings with permanent magnets and a commutator-type rotor. It was constructed under the assumptions adopted for the method of the tooth 
contours conductivities [2]. The authors also assume that the magnetic return parameter of the hard - magnetic material is linear.

The authors assume that each tooth of the stator has one coil (Fig.1). The numeration of the stator teeth and the rotor poles are growing in the direction of the increasing of rotor rotation angle $\gamma$. The current of the tooth contour for singletooth windings is equal to the coil total current of the corresponding tooth $F_{s m}=i_{k m} w_{k}$ (here, $m$ is a tooth number). The magnetic resistances of the sections of the stator magnetic core are taken into account by nonlinear conductivities $\lambda_{j s}$ and $\lambda_{z s}$. The consideration of the windings leakage fluxes is carried out by leakage conductivities $\lambda_{\sigma s}$, which include the slot leakage conductivity. The conductivities between the tooth contours of the stator and the rotor are calculated under special boundary conditions from the field computation in the initial region. The computation can be made by any numerical or analytical approach $[4,5]$.

The permanent magnets, included in the rotor magnetic system will be changed by equivalent single-turn circuits, powered by the current supply with the value equal to the magnet MMF $F_{M 0}$. The MMF is defined by an intersecting point of the return line with the axis of abscissa $H_{m 0}$ and magnet height $h_{m} ; F_{M 0}=H_{m 0} h_{m}$. The configuration and arrangement on the inductor of such circuit for commutatortype rotors coincide with the configuration and arrangement of the replaced magnet. The introduction of the fictitious MMF concept allows one to replace the permanent magnet to the MMF supply with an internal conductivity, equal to the magnetic reluctance of the magnet.

\section{THE SYSTEM OF EQUATION OF THE MAGNETIC CIRCUIT.}

According to the obtained substitution scheme, it is possible to calculate the magnetic fluxes of the circuits and the potentials of the stator and rotor teeth. The following conclusions are based on work [2,3,7,8].

Based on the law of the total current, let us write the following equations for the contours of the stator and rotor, which include two adjacent teeth (poles) and a section of the yokes between them:

$$
\begin{aligned}
& \varphi_{s m}-\varphi_{s, m+1}+U_{m s}-\left(F_{s m}-F_{s, m+1}\right)=0 \\
& \varphi_{R k}-\varphi_{R, k+1}+U_{m R}-F_{M 0}=0
\end{aligned}
$$

where $\varphi_{s m}, \varphi_{s, m+1}, \varphi_{R k}, \varphi_{R, k+1} \quad$ - the scalar magnetic potentials of the teeth, $U_{m s}=\int H_{m} d l$ - drop of the magnetic potential in the stator core, $U_{m R}=\Phi_{R k}\left(1 / \lambda_{M}\right)$ - drop of the magnetic potential in the permanent magnet, $F_{s m}, F_{s, m+1}$ - the stator teeth MMF, $F_{M 0}$ - the magnet MMF.
Let us combine the potentials of the teeth, the MMFs and the drops of the magnetic potential into the corresponding vectors for the stator and rotor:

$$
\begin{aligned}
& \bar{\varphi}_{s}=\left[\varphi_{s 1}\left|\varphi_{s 2}\right| \ldots\left|\varphi_{s m}\right| \ldots\left|\varphi_{s, z 1}\right|\right]^{T} \\
& \bar{\varphi}_{R}=\left[\varphi_{R 1}\left|\varphi_{R 2}\right| \ldots\left|\varphi_{R k}\right| \ldots\left|\varphi_{R, z 2}\right|\right]^{T} \\
& \bar{U}_{m s}=\left[U_{m 1}\left|U_{m 2}\right| \ldots\left|U_{m m}\right| \ldots\left|U_{m, z 1}\right|\right]^{T} \\
& \bar{U}_{m R}=\left[U_{m 1}\left|U_{m 2}\right| \ldots\left|U_{m k}\right| \ldots\left|U_{m, z 2}\right|\right]^{T} \\
& \bar{F}_{s}=\left[F_{s 1}\left|F_{s 2}\right| \ldots\left|F_{s m}\right| \ldots\left|F_{s, z 1}\right|\right]^{T} \\
& \bar{F}_{M 0}=\left[F_{M 0}\left|-F_{M 0} \ldots\right| F_{M 0}||-F_{M 0}\right]^{T}
\end{aligned}
$$

and write (1) for all circuits of the stator and rotor in the matrix form:

$$
\begin{aligned}
& \bar{U}_{m s}+[D]_{S} \bar{\varphi}_{s}-[D]_{S} \bar{F}_{s}=0 \\
& \bar{U}_{m R}+[D]_{R} \bar{\varphi}_{R}-\bar{F}_{M 0}=0
\end{aligned}
$$

Here $[D]_{S},[D]_{R}$ - the matrix transformations which have the following form:

$$
[D]_{S, R}=\left[\begin{array}{rrrrrrr}
-1 & 1 & 0 & 0 & \ldots & 0 & 0 \\
0 & -1 & 1 & 0 & \ldots & 0 & 0 \\
\ldots & \ldots & \ldots & \ldots & \ldots & \ldots & \ldots \\
0 & 0 & 0 & 0 & \ldots & -1 & 1 \\
1 & 0 & 0 & 0 & \ldots & 0 & -1
\end{array}\right] Z_{S(R)}(4)
$$

Solution of (3) with respect to the potentials can be obtained using matrix $[M]$ :

$$
[M]=\left[\begin{array}{c|ccc}
0 & 0 & \ldots & 0 \\
\hline 0 & & \\
\cdots & & {[D]_{1}^{-1}} & \\
0 & & &
\end{array}\right] .
$$

Matrix $[D]_{1}$ is obtained by deleting the first column and the first row of matrix $[D]$.

After the transformations, one will calculate the teeth potentials:

$$
\begin{aligned}
& \bar{\varphi}_{s}=[M]_{S}\left([D]_{S} \bar{F}_{s}-\bar{U}_{m s}\right) \\
& \bar{\varphi}_{R}=[M]_{R}\left(\bar{F}_{M 0}-\bar{U}_{m R}\right)
\end{aligned}
$$

When determining the potentials of the teeth, the condition that the flux should be zero through the air gap of all contours must be taken into account [5]: 


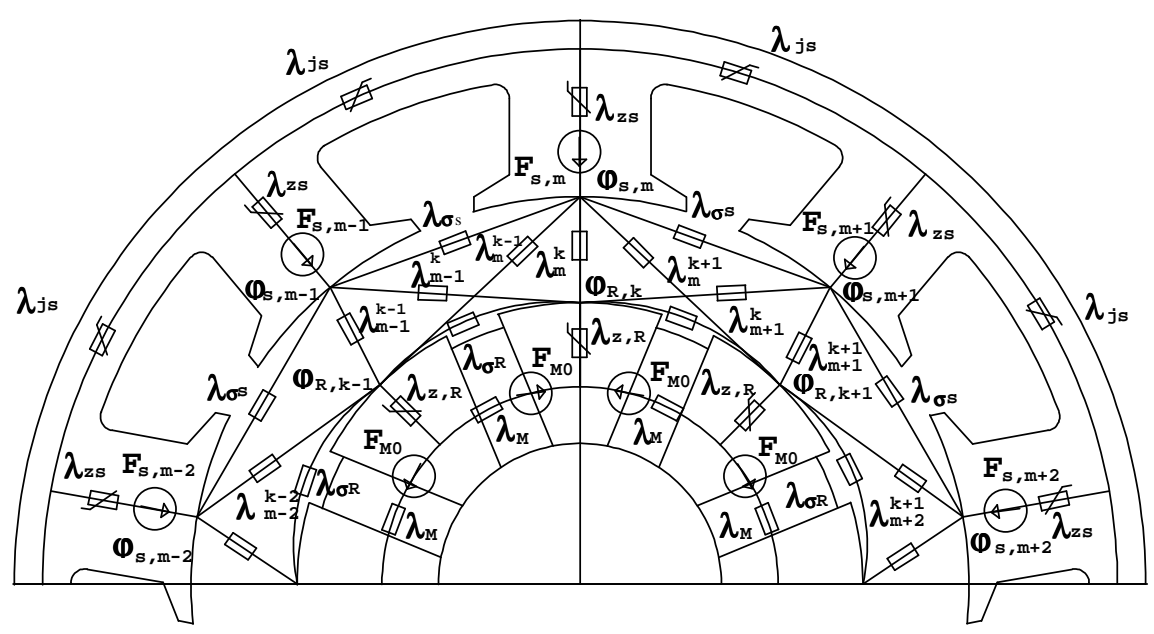

Fig. 1. The equivalent scheme of the magnetic circuit of the machine with with commutator-type rotor.

$$
\sum_{k=1}^{z} \Phi_{k}=0 \quad \text { or } \quad \sum_{k=1}^{z} \varphi_{k} \lambda_{k}=0
$$

where $\lambda_{k}$ - the air gap conductivity for the $k$-th circuit.

As shown in [2], from this equation it follows that the magnitude of the potential of each tooth should differ by some value equal to all teeth:

$$
\varphi_{\delta}=\varphi+\Delta \varphi
$$

where $\Delta \varphi$ is calculated as follows:

$$
\Delta \varphi=-\frac{\sum_{k=1}^{z} \varphi_{k} \lambda_{k}}{\sum_{k=1}^{z} \lambda_{k}} .
$$

Let us write the complete matrix of the conductivities of the magnetic system. The matrix is more conveniently represented as consisting of 4 blocks:

$$
[\lambda]=\left[\begin{array}{l|l}
{[\lambda]_{S}} & {[\lambda]_{S R}} \\
\hline[\lambda]_{R S} & {[\lambda]_{R}}
\end{array}\right]
$$

$[\lambda]_{S}$ - the matrix of conductivities of the self- and mutual inductances of the stator circuits (the subscripts are the numbers of the rotor teeth; the superscripts are the numbers of stator teeth, having mutual conductivity with the said teeth (slot leakage)),

$[\lambda]_{R}$ - the matrix of conductivities of the self and mutual inductance of the rotor circuits (the subscripts are the numbers of the rotor teeth; the superscripts - of the stator teeth);
$[\lambda]_{S R}$ - the matrix of mutual partial conductivities between the stator and rotor circuits (the subscripts are the numbers of the stator teeth; the superscripts are the numbers of the rotor teeth with which the stator teeth have mutual conductivity);

$[\lambda]_{R S}$ - the matrix of mutual partial conductivities between the stator and rotor circuits (the subscripts are the numbers of the rotor teeth; the superscripts are the numbers of the stator teeth with which the rotor teeth have mutual conductivity).

The complete matrix of the magnetic system conductivities is given below.

Let us combine (6) for the stator and the rotor into one equation.

In addition, let us introduce vectors $\bar{\varphi}, \bar{U}_{m}, \bar{F}$, including

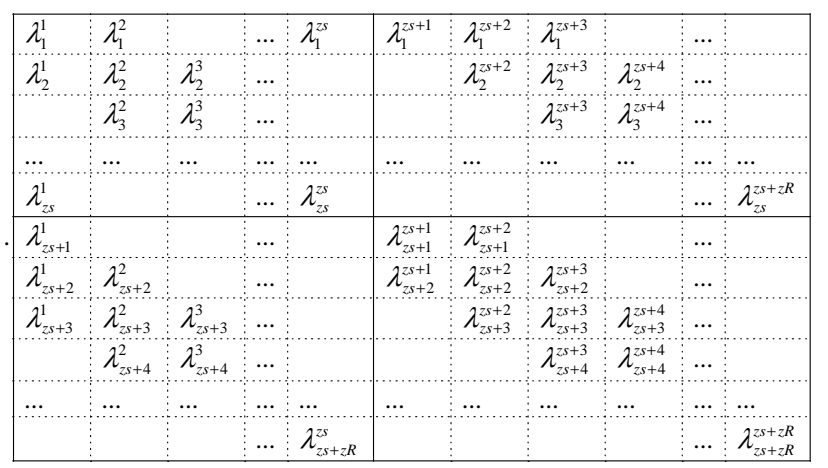

the corresponding vectors of the stator and the rotor. The consecutive numbering from 1 to $\mathrm{ZS}+\mathrm{ZR}$ was introduced for vectors:

$$
\Delta \bar{\varphi}=-\frac{1}{2 \lambda_{c}}[S \llbracket \lambda] \bar{\varphi} .
$$


Here, $\lambda_{c}$ - the unipolar conductivity of the air gap

[S] - the matrix, when one multiplies an arbitrary matrix by it; the elements of the matrix columns are added:

$$
[S]=\left[\begin{array}{ccc|ccc}
1 & \ldots & 1 & & & \\
\ldots & \ldots & \ldots & & 0 & \\
1 & \ldots & 1 & & & \\
\hline & & & 1 & \ldots & 1 \\
& 0 & & \ldots & \ldots & \ldots \\
& & & 1 & \ldots & 1
\end{array}\right] \mathrm{ZS}+\mathrm{ZR}
$$

In view of the above-mentioned information, let us write down, after substituting of $\bar{\varphi}$ in (8) that:

$$
\bar{\varphi}_{\delta}=\left([E]-\frac{1}{2 \lambda_{c}}[S \rrbracket \lambda][M]\left(\bar{F}-\bar{U}_{m}\right) .\right.
$$

Here $[M]$ is composed of $\mathrm{C}$ and $[M]_{R}$ :

$$
[M]=\left[\begin{array}{c|c}
{[M]_{S}} & 0 \\
\hline 0 & {[M]_{R}}
\end{array}\right] .
$$

$\bar{F}$ and $\bar{U}_{m}$ - vectors of the MMF and the drop of potential of the stator and the rotor:

$$
\bar{F}=\left[[D]_{S} \bar{F}_{s} \mid \bar{F}_{M 0}\right], \bar{U}_{m}=\left[\bar{U}_{m s} \mid \bar{U}_{m R}\right] .
$$

The flux vector of the stator and rotor teeth can be determined using the total conductivity matrix and the potential vector of the stator and rotor teeth [6]:

$$
\begin{gathered}
\bar{\Phi}_{z}=[\lambda] \bar{\varphi}_{\delta}, \text { or } \\
\bar{\Phi}_{z}-[\lambda][N]\left(\bar{F}-\bar{U}_{m}\right)=0,
\end{gathered}
$$

here

$$
[N]=\left([E]-\frac{1}{2 \lambda_{c}}[S][\lambda]\right)[M] .
$$

The resulting equation system (14) is nonlinear. It can be solved by the iterative method, for example, by the NewtonRaphson method. In this case, nonlinear conductivities $\lambda_{j s}, \lambda_{z s}, \lambda_{z R}$ of the equivalent network must be calculated in advance and given in the form of dependences $\lambda=f(\Phi)$.

Let us consider the solution of the linear problem in more details. It is possible to assume that the magnetic permeability of the steel is infinite. Then vector $\bar{U}_{m}$ will have the following form:

$$
\bar{U}_{m}=\left[0\left(\frac{1}{\lambda_{M}} \bar{\Phi}_{k R}\right] \text { or } \bar{U}_{m}=[\Lambda]_{M} \Phi_{k},\right.
$$

where

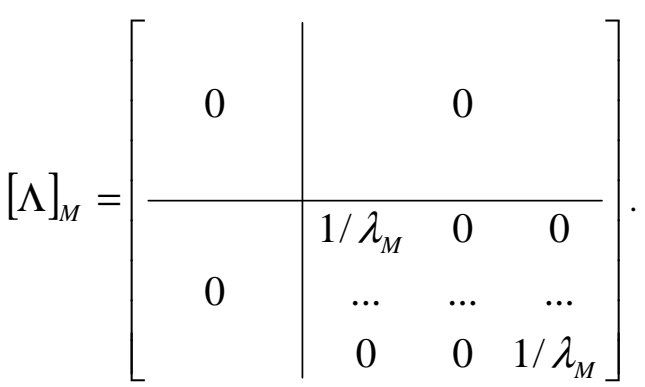

For solving (14), it is necessary to have the values of the circuits' fluxes, which can be calculated from equation:

$$
\begin{aligned}
& \bar{\Phi}_{z s}=[A]_{S} \bar{\Phi}_{k s} \\
& \bar{\Phi}_{z R}=[A]_{R} \bar{\Phi}_{k R}
\end{aligned}
$$

where

$$
[A]=\left[\begin{array}{ccccc}
-1 & 0 & \ldots & 0 & 1 \\
1 & -1 & \ldots & 0 & 0 \\
\cdots & \cdots & \cdots & \cdots & \cdots \\
0 & 0 & \ldots & -1 & 0 \\
0 & 0 & \cdots & 1 & -1
\end{array}\right] \quad \mathrm{ZS} \mathrm{(ZR)}
$$

since $\operatorname{det}[\mathrm{A}]=0,(16)$ do not have solutions in this form.

Let us introduce the condition that the sum of the magnetic potential drops in the circuit, including all the magnets, equals zero:

$$
\sum_{k=1}^{Z R} \frac{1}{\lambda_{M}} \bar{\Phi}_{k R}=0 \text { or } \sum_{k=1}^{Z R} \bar{\Phi}_{k R}=0
$$

This condition is quite strict with the identity of the properties of all the magnets included in the circuit.

From (17), it is possible to express the value of the magnetic flux of the k-th circuit, and then after substitution in (16), one obtains a new equation, which is solvable with respect to $\bar{\Phi}_{k}$ :

$$
[A]_{1 S} \bar{\Phi}_{k s}=\bar{\Phi}_{z s} \text { and }[A]_{1 R} \bar{\Phi}_{k R}=\bar{\Phi}_{z R} .
$$

Here, 


$$
[A]_{1 S(R)}=\left[\begin{array}{rrrrrrr}
-1 & 0 & 0 & \ldots & 0 & 0 & 1 \\
1 & -1 & 0 & \ldots & 0 & 0 & 0 \\
0 & 1 & -1 & \ldots & 0 & 0 & 0 \\
\ldots & \ldots & \ldots & \ldots & \ldots & \ldots & \ldots \\
1 & 1 & 1 & \ldots & 1 & 2 & 0
\end{array}\right]
$$

ZS, ZR

Let us define the contour fluxes:

$$
\bar{\Phi}_{k s}=[A]_{1 S}^{-1} \bar{\Phi}_{z s} \text { and } \bar{\Phi}_{k R}=[A]_{1 R}^{-1} \bar{\Phi}_{z R},
$$

where the inverse matrix has the form of:

$$
[A]_{1}^{-1}=\frac{1}{n}\left[\begin{array}{rrrrrrrr}
0 & n-1 & n-2 & n-3 & \ldots & 3 & 2 & 1 \\
0 & -1 & n-2 & n-3 & \ldots & 3 & 2 & 1 \\
0 & -1 & -2 & n-3 & \ldots & 3 & 2 & 1 \\
\ldots & \ldots & \ldots & \ldots & \ldots & \ldots & \ldots & \ldots \\
0 & -1 & -2 & -3 & \ldots & 3-n & 2 & 1 \\
0 & -1 & -2 & -3 & \ldots & 3-n & 2-n & 1 \\
n & n-1 & n-2 & n-3 & \ldots & 3 & 2 & 1
\end{array}\right] .
$$

Herein for

$$
[A]_{1 S}^{-1}-n=Z S, \text { and for }[A]_{1 R}^{-1}-n=Z R .
$$

Let us combine the fluxes of the stator and rotor circuits into one vector:

$$
\bar{\Phi}_{k}=[B] \bar{\Phi}_{z},
$$

where matrix $[\mathrm{B}]$ is composed of $[A]_{1 S}^{-1} \operatorname{or}[A]_{1 R}^{-1}$ as follows:

$$
[B]=\left[\begin{array}{c|c}
{[A]_{1 S}^{-1}} & 0 \\
\hline 0 & {[A]_{1 R}^{-1}}
\end{array}\right] .
$$
(16):

Let us substitute (20) for the flux of circuits in (15) and

$$
\bar{\Phi}_{z}-[\lambda][N]\left(\bar{F}-[\Lambda]_{M}[B] \Phi_{z}\right)=0,
$$

and solve them with respect to the flux of the teeth:

$$
\left([E]+[\lambda \rrbracket N \rrbracket \Lambda]_{M}[B] \bar{\Phi}_{z}-[\lambda \rrbracket N] \bar{F}=0 .\right.
$$

The obtained system of the linear algebraic equations can be solved with respect to the magnetic fluxes of the teeth by any known method [10].

Given there is a series of values of the angular positions of the rotor and the phase currents, the curves of the flux linkage and emf in time and the electromagnetic torque from the load angle are calculated.
The phase linkages of the phases of a machine with singletooth windings are simply a sum of the teeth fluxes that are in the phase zone.

It is more convenient to calculate the machine torque by the method proposed in [8] by the formula:

$$
M=-\frac{1}{2} \bar{\Phi}_{z}^{t} \frac{d \bar{\varphi}}{d \gamma}
$$

where $\gamma$ - a current rotor angle

$d \bar{\varphi}$ - vector of the potentials increment of the machine tooth circuits during an elementary displacement of the rotor at angle $d \gamma$

When calculating the transient modes, the linkages of the phases can be substituted directly into the equations of electrical equilibrium, and the electromagnetic torque in the equation of torques.

Based on the developed mathematical model of the magnetic circuit calculating, the program for calculating multipole synchronous machines with single-tooth fractional windings with permanent magnets with a commutator-type rotor was created.

\section{CONCLUSIONS}

Based on the method of the tooth contours conductivities, a mathematical model for calculating the magnetic circuit of multipole synchronous machines with fractional single-tooth windings with a commutator-type rotor with permanent magnets has been developed.

The obtained model allows calculating the change in parameters, emf, electromagnetic torque, depending on the rotation angle of the rotor, taking into account two-sided gearing and any form of phase currents.

When adding the equations of electric equilibrium to the obtained model, it is possible to calculate not only steady, but also transient modes of electric machines with permanent magnets.

\section{References}

[1] A.F. Shevchenko, "Magnetomotive forces of one tooth fractional windings with q <1," Nauchnyj Vestnik NGTU, vol. 2 , pp. 99 - 100, 1996.

[2] A.V. Ivanov-Smolensky, Y.V. Abramkin., A.I Vlasov, V.A. Kuznetsov, The universal method of electromagnetic processes in electrical machines calculating, Moscow: Energoatomizdat, 1986.

[3] A.V. Ivanov-Smolensky, B.A. Kuznetsov, "Application of the electric charges technique to the calculation of inductive parameters of electrical machine circuits," Electrichestvo, vol. 1, pp.37-51, 1977.

[4] A.V. Ivanov-Smolensky, M.A. Avanesov, "A calculating method for the unipolar conductivities of tooth contours with allowance for two-sided gearing," [Problems of creating high-torque electromechanical devices Proceedings of MEI, 1980. Publ. 449].

[5] Y.V. Abramkin, A.V. Ivanonv-Smolensky, "The application of the conformed transformation method for the investigation of plane magnetostatic fields in the regions with distributed supplies," Izvestiya Vuzov Electromechanika, vol. 11, 1978. 
[6] V.A. Martyniv, E.K. Sychev, "Mathematical simulation of the fields and processes in the synchronous motors with permanent magnets," Electrichestvo, vol. 3, 1994.

[7] N. Hankok, Matrix analysis of electrical machines, Moscow: Energia, 1967.

[8] A.V. Ivanov-Smolensky, A.I. Vlasov, V.A. Martynov, "Rotating electromagnetic torque of a saturated electric machine," Izvestiya akademii nauk SSSR Energetica i Transport, vol. 4, pp. 74-77, 1983.
[9] A.F. Shevchenko "Multipole synchronous machines with fractional q $<1$ tooth windings with permanent magnets excitation", Elektrotehnika, vol. 9, pp. 3-9, 2007.

[10] I.N. Bornshtein, K.A. Semendyaev, Mathematics Handbook, Moscow: Nauka, 1986. 